TITLE:

\title{
C-13 nuclear Overhauser polarization nuclear magnetic resonance in rotating solids: Replacement of cross polarization in uniformly C-13 labeled molecules with methyl groups
}

\author{
$\operatorname{AUTHOR}(\mathrm{S}):$ \\ Takegoshi, K; Terao, T
}

\section{CITATION:}

Takegoshi, K ...[et al]. C-13 nuclear Overhauser polarization nuclear magnetic resonance in rotating solids: Replacement of cross polarization in uniformly C-13 labeled molecules with methyl groups. JOURNAL OF CHEMICAL PHYSICS 2002, 117(4): 1700-1707

ISSUE DATE:

2002-07-22

URL:

http://hdl.handle.net/2433/49978

\section{RIGHT:}

Copyright 2002 American Institute of Physics. This article may be downloaded for personal use only. Any other use requires prior permission of the author and the American Institute of Physics. 


\title{
${ }^{13} \mathrm{C}$ nuclear Overhauser polarization nuclear magnetic resonance in rotating solids: Replacement of cross polarization in uniformly ${ }^{13} \mathrm{C}$ labeled molecules with methyl groups
}

\author{
K. Takegoshi and Takehiko Terao \\ Department of Chemistry, Graduate School of Science, Kyoto University, Kyoto 606-8502, Japan
}

(Received 21 March 2002; accepted 22 April 2002)

\begin{abstract}
A new ${ }^{13} \mathrm{C}$ polarization technique in solids is presented on the basis of a recently proposed ${ }^{13} \mathrm{C}-{ }^{13} \mathrm{C}$ recoupling sequence $\left[{ }^{13} \mathrm{C}-{ }^{1} \mathrm{H}\right.$ dipolar-assisted rotational resonance (DARR), K. Takegoshi, S. Nakamura, and T. Terao, Chem. Phys. Lett. 344, 631 (2001)] operative under fast magic angle spinning (MAS), in which a rf field is applied to ${ }^{1} \mathrm{H}$ with a rotary resonance condition but none to ${ }^{13} \mathrm{C}$. The ${ }^{1} \mathrm{H}$ irradiation in DARR saturates ${ }^{1} \mathrm{H}$ signals, leading to the ${ }^{13} \mathrm{C}$ signal enhancement due to the nuclear Overhauser effect for fast rotating methyl groups, if any. If we use a uniformly ${ }^{13} \mathrm{C}$ labeled sample, ${ }^{13} \mathrm{C}-{ }^{13} \mathrm{C}$ polarization transfer enhanced by DARR successively distributes the enhanced methyl carbon polarization to the other ${ }^{13} \mathrm{C}$ spins, leading to uniform enhancement for all ${ }^{13} \mathrm{C}$ spins even under very fast MAS. In uniformly ${ }^{13} \mathrm{C}$ labeled rotating samples, the enhancement factor in cross polarization (CP) is about 2.4, while in the present nuclear Overhauser polarization (NOP), it is 3.0 in the fast rotation limit of the methyl groups. While the CP enhancement becomes smaller for molecules with short $T_{1 \rho}$ of ${ }^{1} \mathrm{H}$ or ${ }^{13} \mathrm{C}$, NOP would work well for such mobile molecules, and also NOP enables us to acquire a signal with a short repetition time even if ${ }^{1} \mathrm{H} T_{1}$ is long. Further, NOP has the advantage of quantitativeness, and is very easy to carry out, being insensitive to the adjustment of $\mathrm{rf}$ field intensity and requiring only very low $\mathrm{rf}$ power. These features are demonstrated for uniformly ${ }^{13} \mathrm{C}$, ${ }^{15} \mathrm{~N}$-labeled $L$-threonine and uniformly ${ }^{13} \mathrm{C}$, ${ }^{15} \mathrm{~N}$-labeled glycylisoleucine. NOP-MAS is also applied for a naturally abundant ${ }^{13} \mathrm{C}$ sample.

(C) 2002 American Institute of Physics. [DOI: 10.1063/1.1485062]
\end{abstract}

\section{INTRODUCTION}

Cross polarization $(\mathrm{CP})$ is a prerequisite technique for improving the sensitivity in solid-state NMR of dilute and/or low $\gamma$ spins $(S){ }^{1,2} \mathrm{CP}$ enables us to acquire a signal with a repetition time of the spin-lattice relaxation time $\left(T_{1}\right)$ of the abundant ${ }^{1} \mathrm{H}$ spins $(I)$, which is usually much shorter than those of the $S$ spins. CP also enhances the magnetization of the $S$ spins with a low gyromagnetic ratio by $\sim \gamma_{I} / \gamma_{S}$ compared to the thermal equilibrium value. However, as will be pointed out below, this advantage of $\mathrm{CP}$ becomes less appealing for multiply/uniformly ${ }^{13} \mathrm{C}$-labeled samples. In this work, we examined the possibility of replacing $\mathrm{CP}$ by a polarization technique based on the nuclear Overhauser effect (NOE). ${ }^{3}$

In liquid NMR of low $\gamma$ spins, NOE has widely been used to achieve a short repetition time and signal enhancement. ${ }^{4,5}$ NOE is brought about by rf irradiation capable of saturating ${ }^{1} \mathrm{H}$ spins dipolar coupled to the observed spins. Cross relaxation in the coupled spin system under rf irradiation leads the spin system into a quasiequilibrium state in which the signal intensities are enhanced appreciably. In contrast to the popularity of NOE in liquid NMR, however, application of NOE in high-resolution NMR in solids has not been fully examined yet. This is ascribed to the NOE mechanism unfavorable for rigid molecules. For efficient NOE, (i) the spin to be enhanced should relax dominantly by the fluctuation of the dipolar interaction with the spin under rf irra- diation, and further, (ii) the correlation time of the motion responsible for the dipolar fluctuation should be smaller than the inverse of the Larmor frequency of the irradiated spin. Both conditions are fulfilled for molecules in liquid, however, condition (ii) in particular is not realized for solid molecules in general. Some exceptional examples are found in mobile rubbery polymers. ${ }^{6}$ For a rigid solid, it is envisaged that NOE would be appreciable for molecules with fast internal motion. In fact, Naito et al. examined the spin-lattice relaxation of ${ }^{13} \mathrm{C}$ spins in $L$-alanine with or without ${ }^{1} \mathrm{H} \mathrm{rf}$ irradiation, and found an appreciable NOE signal enhancement for the $\mathrm{CH}_{3}$ carbon. ${ }^{7}$ This is reasonable because ${ }^{13} \mathrm{C}$ relaxation in solids is dominated by the ${ }^{13} \mathrm{C}-{ }^{1} \mathrm{H}$ dipolar coupling, and the $\mathrm{CH}_{3}$ group rotates rapidly around the $\mathrm{C}-\mathrm{CH}_{3}$ bond.

Recently, we developed a novel ${ }^{13} \mathrm{C}-{ }^{13} \mathrm{C}$ polarization transfer method $\left[{ }^{13} \mathrm{C}-{ }^{1} \mathrm{H}\right.$ dipolar-assisted rotational resonance (DARR) ]. ${ }^{8}$ In DARR, the ${ }^{13} \mathrm{C}-{ }^{1} \mathrm{H}$ dipolar interaction is recovered by the ${ }^{1} \mathrm{H}$ rf irradiation with intensity $\nu_{1}$ satisfying the rotary-resonance condition $\nu_{1}=n \nu_{\mathrm{MAS}}(n=1$ or 2), ${ }^{9}$ where $\nu_{\text {MAS }}$ is the spinning frequency. The spectral overlap between the two relevant ${ }^{13} \mathrm{C}$ spins, which is required for efficient polarization transfer based on rotational resonance, ${ }^{10-12}$ is realized between a spinning sideband of one ${ }^{13} \mathrm{C}$ spin and the ${ }^{13} \mathrm{C}-{ }^{1} \mathrm{H}$ dipolar pattern of the other ${ }^{13} \mathrm{C}$ spin and vice versa. In the present work, we examined the possibility of achieving NOE and enhancing the signals of all rigid carbons by DARR. Since we applied rf to ${ }^{1} \mathrm{H}$ spins in 
DARR, NOE enhancement would occur for ${ }^{13} \mathrm{C}$ spins in fast rotating groups such as $\mathrm{CH}_{3}$. The enhanced polarization is then successively transfered by DARR to the other ${ }^{13} \mathrm{C}$, leading to overall NOE enhancement even for stationary ${ }^{13} \mathrm{C}$. For the latter purpose, however, DARR may not be optimal, because it was shown that DARR recoupling occurs bandselectively between carboxyl/carbonyl/aromatic carbons and aliphatic carbons. ${ }^{8}$ We recently found that in the second order, recoupling does occur among ${ }^{13} \mathrm{C}$ resonances with smaller chemical shift differences, such as aliphatic carbons. Although its efficiency is not very high, it is still appreciable for a long recoupling time. Theoretical details will be published elsewhere. Experimentally, it will be shown below that the recoupling occurs nonselectively for a recoupling time of a few seconds. We refer to this method of enhancing signal intensities by NOE and DARR as nuclear Overhauser polarization (NOP).

\section{EXPERIMENT}

Uniformly ${ }^{13} \mathrm{C},{ }^{15} \mathrm{~N}$-labeled $L$-threonine (Thr) was purchased from Cambridge Isotope Laboratories, Inc. and used without purification. Uniformly ${ }^{13} \mathrm{C},{ }^{15} \mathrm{~N}$-labeled glycylisoleucine (Gly-Ile) was prepared as described previously. ${ }^{13}$ The NMR experiments on Thr and Gly-Ile were carried out using a Chemagnetics CMX-400 spectrometer operating at the resonance frequency of $100.3 \mathrm{MHz}$ for ${ }^{13} \mathrm{C}$ with a CP/MAS probe (Chemagnetics) for a $3.2 \mathrm{~mm}$ rotor. Both of the MAS frequency $\nu_{\text {MAS }}$ and the ${ }^{1} \mathrm{H}$ rf intensity $\nu_{1}$ for DARR were 20 $\mathrm{kHz}$. The TPPM decoupling ${ }^{14}$ was used with the nutation angle and the phase-modulation angle being optimized for a rf intensity of $100 \mathrm{kHz}$ to be $180^{\circ}$ and $\pm 15^{\circ}$, respectively. The NMR experiments on nonlabeled dimedone (5,5dimethyl-1,3-cyclohexanedione) were done using a Chemagnetics CMX-300 spectrometer operating at the resonance frequency of $75.6 \mathrm{MHz}$ for ${ }^{13} \mathrm{C}$ with a CP/MAS probe (Doty Sci., Inc.) for a $5 \mathrm{~mm}$ rotor. Both of the MAS frequency and the DARR rf intensity were $10 \mathrm{kHz}$, and the $\mathrm{CW}{ }^{13} \mathrm{C}-{ }^{1} \mathrm{H}$ decoupling was adopted with an intensity of $\approx 80 \mathrm{kHz}$.

For CP enhancement at $\nu_{\mathrm{MAS}}=20 \mathrm{kHz}$, the ${ }^{1} \mathrm{H}$ rf intensity was $50 \mathrm{kHz}$ and the ${ }^{13} \mathrm{C}$ rf intensity was varied linearly from 68 to $72 \mathrm{kHz}$ with the contact time devided into 10 segments during each of which the ${ }^{13} \mathrm{C}$ rf intensity was kept constant. The signal enhancement based on NOP was examined as follows: Prior to ${ }^{1} \mathrm{H}$ irradiation, three $90^{\circ}$ pulses were applied to ${ }^{13} \mathrm{C}$ with an interval of $10 \mathrm{~ms}$ to remove ${ }^{13} \mathrm{C}$ longitudinal magnetization. Then a ${ }^{1} \mathrm{H}$ rf field is applied for a certain NOP time, and the resultant magnetization is observed under ${ }^{1} \mathrm{H}$ decoupling. The ${ }^{1} \mathrm{H}$ rf-field strength for NOP was set to satisfy the DARR condition $\left(\nu_{1}=\nu_{\text {MAS }}\right)$. The ${ }^{13} \mathrm{C}$ spin-lattice relaxation curves were observed using the Torchia's pulse sequence. ${ }^{15}$

\section{THEORY}

First, we compare the possible enhancement factors for $\mathrm{CP}$ and NOE. The enhancement factor of $\mathrm{CP}$ is given by ${ }^{1,2}$

$$
\eta_{\mathrm{CP}}=\frac{\gamma_{I}}{\gamma_{S}} \frac{N_{I}}{N_{I}+N_{S}},
$$

where $N_{I}$ and $N_{S}$ are the numbers of $I$ and $S$ spins, respectively. The average proton to carbon ratio $N_{I} / N_{S}$ is calculated to be 1.65 for the 20 standard amino-acid residues in a peptide, leading to the theoretical maximum gain of $\approx 2.4$ for a fully ${ }^{13} \mathrm{C}$-labeled peptide. For a dipolar coupled two-spin $(I-S)$ system, Solomon derived simultaneous differential equations, ${ }^{16}$ which was further extended to a system with a dilute $S$ spin in abundant $I$ spins having a common spin temperature. ${ }^{17}$ Under the steady-state condition with ${ }^{1} \mathrm{H}$ saturation, the NOE factor can formally be written as

$$
\xi_{\mathrm{NOE}}=\frac{\gamma_{I}}{\gamma_{S}} \frac{6 J\left(\omega_{I}+\omega_{S}\right)-J\left(\omega_{I}-\omega_{S}\right)}{J\left(\omega_{I}-\omega_{S}\right)+3 J\left(\omega_{S}\right)+6 J\left(\omega_{I}+\omega_{S}\right)},
$$

where $J(\omega)$ is a spectral density function, and $\omega_{X}$ is the Larmor frequency of the $X$ spin. For a rotating methyl group, Naito et al. have deduced an apparent form of $J(\omega) ;^{7}$ however, the following form is sufficient for evaluation of $\xi_{\mathrm{NOE}}$ :

$$
J(\omega) \propto \frac{\tau_{r}}{1+\omega^{2} \tau_{r}^{2}},
$$

where $\tau_{r}$ is the rotational correlation time $\tau$ of the $\mathrm{CH}_{3}$ group. Note here that the overall signal intensity for ${ }^{13} \mathrm{C}$ becomes $1+\xi_{\mathrm{NOE}}$ times of that obtained at thermal equilibrium and we define the NOE enhancement factor $\eta_{\mathrm{NOE}}$ as $1+\xi_{\mathrm{NOE}}$ to compare directly with $\eta_{\mathrm{CP}}$. The enhancement reaches the maximum,

$$
\eta_{\mathrm{NOE}}=1+\frac{1}{2} \frac{\gamma_{I}}{\gamma_{S}}
$$

at the extreme narrowing limit of relaxation, i.e., $J(\omega)$ $\sim \tau_{r}$. For $I={ }^{1} \mathrm{H}$ and $S={ }^{13} \mathrm{C}$, this becomes ca. 3.0. Hence, the maximum intensity possibly achieved by NOP is somewhat better than that by $\mathrm{CP}$ for fully ${ }^{13} \mathrm{C}$-labeled peptides.

The polarization created for ${ }^{13} \mathrm{CH}_{3}$ is then distributed to the other ${ }^{13} \mathrm{C}$ spins by DARR recoupling. To incorporate the ${ }^{13} \mathrm{C}$ spins coupled to ${ }^{13} \mathrm{CH}_{3}$ in the calculation, we consider a spin system consisting of $N L_{i}\left({ }^{13} \mathrm{C}\right)$ spins $(i=1, \ldots, N)$ coupled to the $S\left({ }^{13} \mathrm{C}\right)$ spin of a rotating $\mathrm{CH}_{3}$ group. For simplicity, we assume that there is no cross relaxation between the ${ }^{1} \mathrm{H}$ spins of the $\mathrm{CH}_{3}$ group $(I)$ and the $L_{i}$ spins. Similar to the assumption made by Brooks et al., ${ }^{17}$ the other ${ }^{1} \mathrm{H}$ spins are assumed to have the common spin temperature and are not apparently included. In other words, we assume that only the ${ }^{13} \mathrm{C}$ spin of the $\mathrm{CH}_{3}$ group is enhanced by NOE due to the $\mathrm{CH}_{3}$ protons. Experimentally, however, we found the direct NOE takes place for ${ }^{13} \mathrm{C}$ spins in close proximity to $\mathrm{CH}_{3}$ groups as will be shown below. Here we would like to examine effects of the presence of the other ${ }^{13} \mathrm{C}$ spins to $\eta_{\mathrm{NOP}}$ quantitatively.

The simultaneous differential equations describing the time dependence of the magnetizations are written as

$$
\frac{d\left\langle I_{Z}\right\rangle}{d t}=-\frac{1}{T_{1}^{I}}\left(\left\langle I_{Z}\right\rangle-I_{0}\right)-\frac{1}{T_{1}^{I S}}\left(\left\langle S_{Z}\right\rangle-S_{0}\right)-R\left\langle I_{Z}\right\rangle,
$$




$$
\begin{aligned}
\frac{d\left\langle S_{Z}\right\rangle}{d t}= & -\frac{1}{T_{1}^{S}}\left(\left\langle S_{Z}\right\rangle-S_{0}\right)-\frac{1}{T_{1}^{S I}}\left(\left\langle I_{Z}\right\rangle-I_{0}\right) \\
& -\sum_{i=1}^{N} k_{i}\left(\left\langle S_{Z}\right\rangle-\left\langle L_{i Z}\right\rangle\right), \\
\frac{d\left\langle L_{i Z}\right\rangle}{d t}= & -\frac{1}{T_{1}^{L_{i}}}\left(\left\langle L_{i Z}\right\rangle-L_{0}\right)-k_{i}\left(\left\langle L_{i Z}\right\rangle-\left\langle S_{Z}\right\rangle\right),
\end{aligned}
$$

where $k_{i}$ is the polarization transfer rate between $S$ and $L_{i}$, $T_{1}^{X}$ is the spin-lattice relaxation time of the $X$ spin, $T_{1}^{X Y}$ is the cross relaxation time between the $X$ and $Y$ spins, and $X_{0}$ denotes the thermal-equilibrium magnetization of the $X$ spin. The last term in Eq. (5) represents the saturation effect due to ${ }^{1} \mathrm{H}$ rf irradiation. We further assume that the polarization transfer rates for the $L_{i}$ spins are equal $\left(k_{i}=k\right)$ and also the relaxation time $\left(T_{1}^{L_{i}}=T_{1}^{L}\right)$. When ${ }^{1} \mathrm{H}$ is saturated $(R$ $\gg 1 / T_{1}^{I}, 1 / T_{1}^{I S}$ and thus $\left.\left\langle I_{Z}\right\rangle=0\right)$ and the system reaches the internal equilibrium, we have

$$
\begin{aligned}
\left\langle S_{Z}\right\rangle_{\mathrm{eq}} & =\left\{1+\frac{1+k T_{1}^{L}}{1+k\left(T_{1}^{L}+N T_{1}^{S}\right)} \xi_{\mathrm{NOE}}\right\} S_{0} \\
& \equiv \eta_{\mathrm{NOP}}^{S} S_{0}, \\
\left\langle L_{Z}\right\rangle_{\mathrm{eq}} & =\left\{1+\frac{k T_{1}^{L}}{1+k\left(T_{1}^{L}+N T_{1}^{S}\right)} \xi_{\mathrm{NOE}}\right\} L_{0} \\
& \equiv \eta_{\mathrm{NOP}}^{L} L_{0},
\end{aligned}
$$

where $\left\langle L_{Z}\right\rangle$ is the average of the $z$ magnetization of the $L_{i}$ magnetizations $\left\langle L_{Z}\right\rangle=(1 / N) \Sigma_{i}\left\langle L_{i Z}\right\rangle, \eta_{\mathrm{NOP}}^{X}$ is the NOP enhancement factor for the $X$ spin, and we used the relation, ${ }^{16}$

$$
\xi_{\mathrm{NOE}}=\frac{T_{1}^{S}}{T_{1}^{S I}} \frac{I_{0}}{S_{0}}
$$

which is identical to Eq. (2) when the relaxation times are governed by the ${ }^{13} \mathrm{C}-{ }^{1} \mathrm{H}$ dipolar fluctuation. Note that for the slow polarization transfer limit $k \sim 0$, the NOP enhancement factor for the $S$ spin is identical to the NOE enhancement factor $\eta_{\mathrm{NOE}}=1+\xi_{\mathrm{NOE}}$, while that for the $L$ spin is $\eta_{\mathrm{NOP}}^{L}$ $=1$. On the other hand, at the fast transfer limit $\left(k T_{1}^{L}\right.$ $\gg 1, k N T_{1}^{S}$ ), both NOP enhancement factors become equal to be $\eta_{\mathrm{NOE}}$. At the intermediate region, Eq. (8) indicates a reduced enhancement factor for $\eta_{\mathrm{NOP}}^{S}$ as compared to $\eta_{\mathrm{NOE}}$ due to the presence of the $L$ spins and further the NOP enhancement factor for the $L$ spins is smaller than that for the $S$ spin.

\section{RESULTS AND DISCUSSION}

First, better enhancement by NOP is demonstrated for a powder sample of uniformly ${ }^{13} \mathrm{C},{ }^{15} \mathrm{~N}$-labeled $L$-threonine (Thr). Figure 1 shows the ${ }^{13} \mathrm{C}$ MAS spectra obtained for Thr using a single $90^{\circ}$ pulse without NOP (a), with NOP (b), and using CP (c). 64 FIDs were accumulated for each experiment, and the spectra in Fig. 1 are plotted on the same amplitude scale and can be directly compared. For the single $90^{\circ}$ pulse experiment with NOP [Fig. 1(b)], the NOP time was $10 \mathrm{~s}$ with the ${ }^{1} \mathrm{H}$ rf-intensity fulfilling the DARR condi-
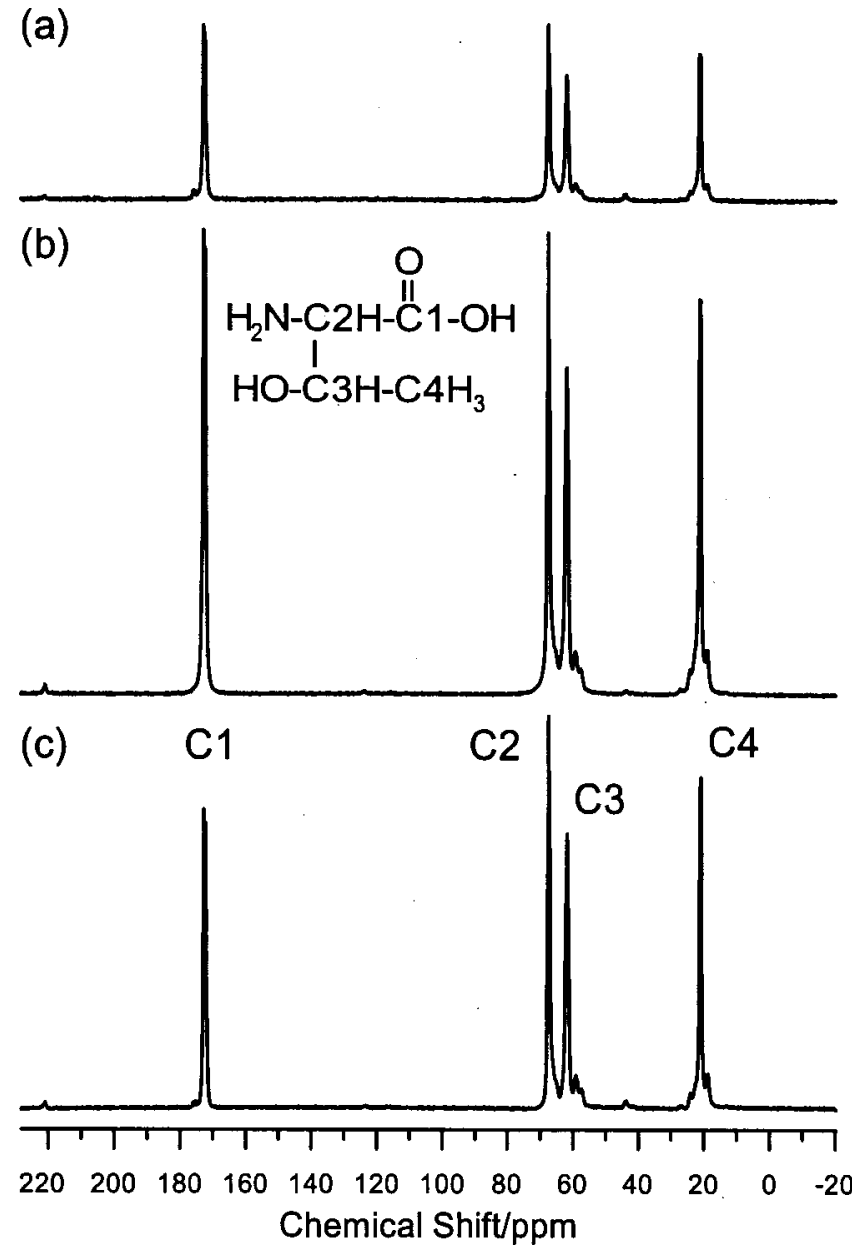

FIG. $1 .{ }^{13} \mathrm{C}$ MAS spectra of uniformly ${ }^{13} \mathrm{C},{ }^{15} \mathrm{~N}$-labeled L-threonine observed using a ${ }^{13} \mathrm{C} 90^{\circ}$ pulse without NOP (a) and with NOP (b) and using $\mathrm{CP}$ (c). For the ${ }^{13} \mathrm{C}$ pulse experiment without NOP, the relaxation interval was $300 \mathrm{~s}$. For NOP, the NOP time was $10 \mathrm{~s}$. For CP, the relaxation interval was $5 \mathrm{~s}$ and the $\mathrm{CP}$ contact time was $3.8 \mathrm{~ms}$. 64 FIDs were accumulated for each experiment, and the spectra are plotted on the same amplitude scale and can be directly compared.

tion $\left(\nu_{1}=\nu_{\mathrm{MAS}}=20 \mathrm{kHz}\right)$. For the $\mathrm{CP}$ spectrum [Fig. 1(c)], the relaxation interval of $5 \mathrm{~s}\left({ }^{1} \mathrm{H} T_{1} \sim 0.8 \mathrm{~s}\right)$ and the $\mathrm{CP}$ contact time of $3.8 \mathrm{~ms}$ were used. Since the repetition time of $300 \mathrm{~s}$ for the single $90^{\circ}$ pulse experiment [Fig. 1(a)] is much longer than the longest ${ }^{13} \mathrm{C} T_{1}$ value $(\sim 30 \mathrm{~s})$ in $\mathrm{Thr}$, the spectrum ensures the full signal intensities at thermal equilibrium. We then obtained the enhancement factor in NOP $\left(\eta_{\mathrm{NOP}}\right)$ and that in $\mathrm{CP}\left(\eta_{\mathrm{CP}}\right)$; the area intensity of each peak in Figs. 1(b) and 1(c) is expressed in values relative to the corresponding intensity in Fig. 1(a). The obtained enhancement factors in NOP are $\eta_{\mathrm{NOP}}=2.65,2.67,2.66$, and 2.72 for $\mathrm{C} 1(\mathrm{COOH}), \mathrm{C} 2\left(\mathrm{C}_{\alpha}\right), \mathrm{C} 3\left(\mathrm{C}_{\beta}\right)$, and $\mathrm{C} 4\left(\mathrm{CH}_{3}\right)$, respectively, which are significantly larger than those in $\mathrm{CP}: \eta_{\mathrm{CP}}$ $=1.52,1.99,2.04$, and 2.02 , for $\mathrm{C} 1-\mathrm{C} 4$, respectively. All four ${ }^{13} \mathrm{C}$ signals are almost uniformly enhanced by NOP, but not by $\mathrm{CP}$. The unequal enhancement by $\mathrm{CP}$ is attributed mainly to different optimal CP contact times and different ${ }^{13} \mathrm{C}$ spin-lattice relaxation times $\left(T_{1 \rho}^{\mathrm{S}}\right)$ in the rotating frame for different carbons. In fact, it was observed that an optimal $\mathrm{CP}$ contact time for $\mathrm{C} 2-\mathrm{C} 4$ is $\approx 0.8 \mathrm{~ms}$, while that for $\mathrm{C} 1$ is $\approx 3.8 \mathrm{~ms}$. Even at these optimal contact times for the indi- 
vidual carbons, however, we observed unequal $\eta_{\mathrm{CP}}$ values: $C 1=1.5, C 2=2.2, C 3=2.1$, and $C 4=2.4$ (spectra not shown). This fact shows that even if the optimal contact times are equal for all carbons, quantitative comparison of signal intensities are difficult due to different $T_{1 \rho}^{\mathrm{S}}$.

The rotational correlation time of the $\mathrm{CH}_{3}$ group at 300 $\mathrm{K}$ is calculated to be $\tau_{r}=6.1 \times 10^{-11} \mathrm{~s}$ from the activation energy and the pre-exponential factor reported for L-threonine. ${ }^{18}$ For this $\tau_{r}$ value, the NOE enhancement factor is calculated from Eq. (2) to be $\eta_{\mathrm{NOE}} \sim 2.9$, which is in agreement with that observed $\left(\eta_{\mathrm{NOP}} \sim 2.7\right)$ when the reduction indicated in Eq. (8) is taken into account. For example, putting $N=3$ for $\mathrm{Thr}$ and $T_{1}^{L}=10 \mathrm{~s}, T_{1}^{S}=0.25 \mathrm{~s}$, and $k$ $=1 \mathrm{~s}^{-1}$, which were estimated roughly for Thr, into Eq. (8), we have $\eta_{\mathrm{NOP}}^{S} \sim 2.8$. Further, the somewhat smaller $\eta_{\mathrm{NOP}}$ for the other carbons $(\mathrm{C} 1-\mathrm{C} 3)$ can also be explained by Eq. (8) for a finite $k$ value in the intermediate region. The NOP enhancement in Thr is efficient owing to the large NOE due to the short rotational correlation time of the $\mathrm{CH}_{3}$ group and also owing to the fast ${ }^{13} \mathrm{C}-{ }^{13} \mathrm{C}$ transfer due to the short ${ }^{13} \mathrm{C}-{ }^{13} \mathrm{C}$ distances between the $\mathrm{CH}_{3}$ carbon and the other three carbon atoms.

Next, we undertook NOP experiments of uniformly ${ }^{13} \mathrm{C}$, ${ }^{15} \mathrm{~N}$-labeled glycylisoleucine(Gly-Ile), of which the two carbons of the glycine are far from the two $\mathrm{CH}_{3}$ carbons of the isoleucine sidechain. Figures 2(a) and 2(b) show the normalized ${ }^{13} \mathrm{C}$ spectra observed for Gly-Ile with a repetition time of $5 \mathrm{~s}$ (a) without NOP and (b) with NOP (DARR at $\nu_{1}$ $=\nu_{\mathrm{MAS}}=20 \mathrm{kHz}$ ), showing that appreciable NOP enhancement occurs uniformly for all eight carbons whose assignment is given in Ref. 13 . This shows that the ${ }^{13} \mathrm{C}-{ }^{13} \mathrm{C}$ transfer under DARR is sufficiently fast to distribute the ${ }^{13} \mathrm{C}$ polarization created by NOP for the ${ }^{13} \mathrm{CH}_{3}$ carbons $(\mathrm{C} 7, \mathrm{C} 8)$ in the isoleucine sidechain to the other carbons. The enhanced ${ }^{13} \mathrm{C}-{ }^{13} \mathrm{C}$ transfer by DARR irradiation was appreciated further by observing ${ }^{13} \mathrm{C}$ spin-lattice relaxation curves. Figures 3(a) and 3(b) show the ${ }^{13} \mathrm{C}$ spin-lattice relaxation curves of Gly-Ile with (a) and without (b) the DARR irradiation $\left(\nu_{1}=\nu_{\mathrm{MAS}}=20 \mathrm{kHz}\right)$. The curves observed without DARR [Fig. 3(b)] show that the ${ }^{13} \mathrm{C}$ magnetizations decay with the individual time constants of $\approx 0.1-30 \mathrm{~s}$. The time constants spread in a wide range demonstrates that the ${ }^{13} \mathrm{C}-{ }^{13} \mathrm{C}$ polarization transfer (spin diffusion) among them is considerably slow. This is brought about by averaging of ${ }^{13} \mathrm{C}-{ }^{13} \mathrm{C}$ dipolar couplings by fast MAS $\left(\nu_{\mathrm{MAS}}=20 \mathrm{kHz}\right)$. Analysis of the ${ }^{13} \mathrm{C} T_{1}$ curves to deduce polarization transfer rates would be interesting, however, it is out of the scope of this work, and will be published elsewhere. The curves under DARR [Fig. 3(a)] show that the polarization transfer rates turn significantly higher under DARR, allowing the ${ }^{13} \mathrm{C}$ spins with short $T_{1}$ to act as a sink of relaxation. Similar equalization of $T_{1}$ by fast spin diffusion is commonly found for ${ }^{1} \mathrm{H}$ spins in solids, ${ }^{19,20}$ which has been taken as a direct evidence for the legitimacy of the spin-temperature hypothesis. For ${ }^{13} \mathrm{C}$ spins under DARR, however, Fig. 3(a) shows that the ${ }^{13} \mathrm{C}-{ }^{13} \mathrm{C}$ polarization transfer is much enhanced, but is still not fast enough to achieve a common spin temperature within 1-2 s. Nevertheless it occurs nonselectively, indicat-

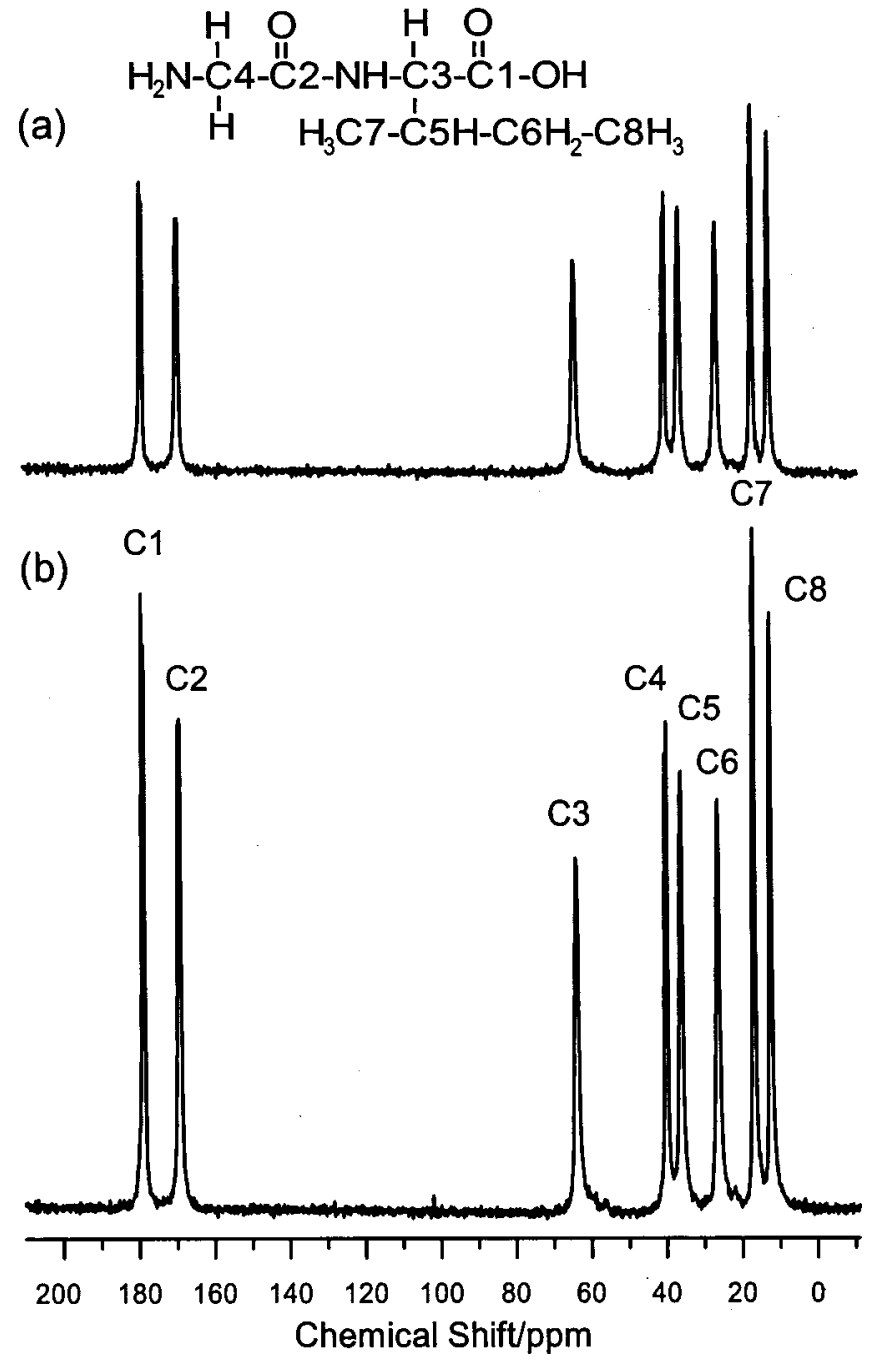

FIG. 2. ${ }^{13} \mathrm{C}$ MAS spectra of uniformly ${ }^{13} \mathrm{C},{ }^{15} \mathrm{~N}$-labeled glycylisoleucine observed by a ${ }^{13} \mathrm{C} 90^{\circ}$ pulse (a) without DARR irradiation and the pulse repetition time of $5 \mathrm{~s}$ and (b) with DARR irradiation for $5 \mathrm{~s}$. 64 FIDs were accumulated for each experiment, and the two spectra are plotted on the same amplitude scale and can be directly compared.

ing the capability of distributing the polarization created by NOE in ${ }^{13} \mathrm{CH}_{3}$ to the other ${ }^{13} \mathrm{C}$ spins.

Figures 4(a) and 4(b) show the NOP-time dependence of the signal area intensities for the eight carbons of Gly-Ile with (a) the ${ }^{1} \mathrm{H}$ rf-intensity at the DARR condition $\left(\nu_{1}\right.$ $\left.=\nu_{\mathrm{MAS}}=20 \mathrm{kHz}\right)$ and (b) that at the off-DARR condition of $\nu_{1}=6 \mathrm{kHz}$, which may be enough to cause NOE but ${ }^{13} \mathrm{C}-{ }^{13} \mathrm{C}$ recoupling efficiency is low. The signal intensities for the individual carbons at various NOP times are normalized by the signal intensities observed using a ${ }^{13} \mathrm{C} 90^{\circ}$ pulse with a repetition time of $300 \mathrm{~s} \geqslant 5 T_{1}$. Hence, the final intensities represent the NOP enhancement factors $\eta_{\mathrm{NOP}}$ for the individual carbons. It was observed that the time required for all carbons other than the two methyl carbons to reach an equilibrated intensity is shorter for the experiment under DARR [Fig. 4(a)] than that under off-DARR irradiation [Fig. 4(b)], which is most apparently appreciated for $\mathrm{C} 2\left({ }^{13} \mathrm{C}=\mathrm{O}\right.$ of Gly) and $\mathrm{C} 4\left({ }^{13} \mathrm{CH}_{2}\right.$ of Gly). The slower equilibration rates observed for them under off-DARR irradiation [Fig. 4(b)] are ascribed to the fact that these two carbons are dis- 
(a)

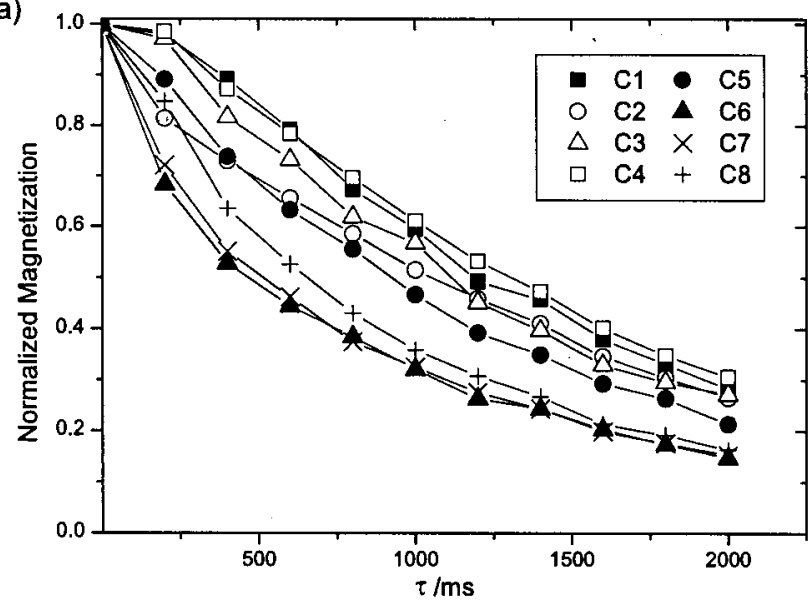

(b)

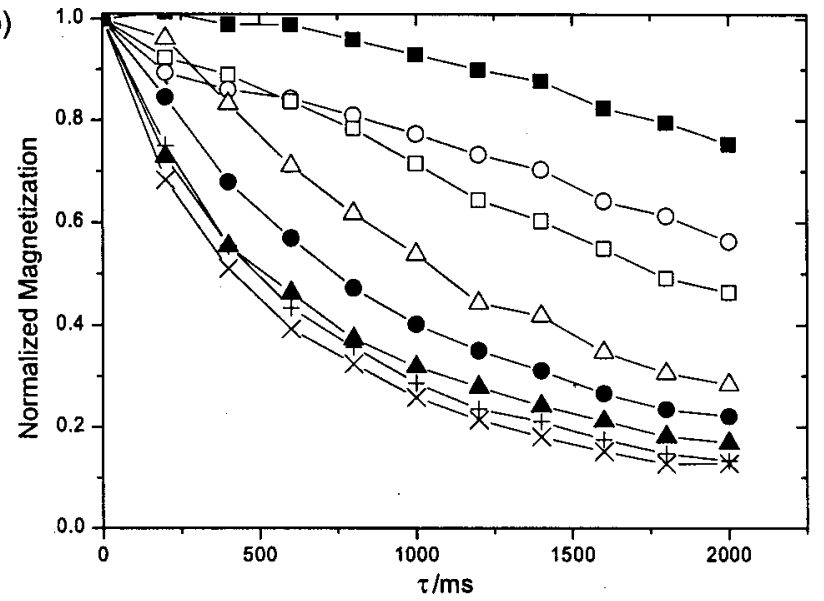

FIG. $3 .{ }^{13} \mathrm{C}$ spin-lattice relaxation curves of uniformly ${ }^{13} \mathrm{C},{ }^{15} \mathrm{~N}$-labeled glycylisoleucine with (a) and without (b) the DARR irradiation. The Torchia's pulse sequence (Ref. 15) was used under $\nu_{\mathrm{MAS}}=20 \mathrm{kHz}$. The assignment of the ${ }^{13} \mathrm{C}$ signals numbered in Fig. 2(b) is found in Ref. 13. The solid lines are for eye guidance.

tant from either of the two $\mathrm{CH}_{3}$ carbons ( $\mathrm{C} 7$ and $\mathrm{C} 8$ of Ile).

In Table I, we collate the ${ }^{13} \mathrm{C}-{ }^{13} \mathrm{C}$ distances from the methyl carbons $\left(r_{\mathrm{C}-\mathrm{C} 7,8}\right)$, which are calculated from the atomic coordinates determined by an x-ray diffraction study (unpublished). Table I also lists the apparent NOP buildup rates $k_{\mathrm{NOP}}$ determined by fitting the observed data to the following single exponential function,

$$
S(t) / S_{0}=\eta_{\mathrm{NOP}}\left\{1-\exp \left(-k_{\mathrm{NOP}} t\right)\right\}
$$

using $\eta_{\mathrm{NOP}}$ and $k$ as fitting parameters. The solid curves in Fig. 4 are the best-fit curves with the parameters listed in Table I. It should also be pointed out here that Eq. (10) is derived from Eq. (6) at the slow polarization transfer limit $(k=0)$ with $k_{\mathrm{NOP}}=1 / T_{1}^{S}$ under ${ }^{1} \mathrm{H}$ saturation $\left(\left\langle I_{Z}\right\rangle=0\right)$. Due mostly to these simplifying assumptions, the singleexponential fitting does not reproduce the observation particularly at the initial NOP region. We have not tried to fit the observed data by using Eqs. (5)-(7) because of the complexity of the eight ${ }^{13} \mathrm{C}$ spin system.

Enhanced ${ }^{13} \mathrm{C}-{ }^{13} \mathrm{C}$ transfer is evidently observed for $\mathrm{C} 1-\mathrm{C} 4, k_{\mathrm{NOP}}$ under DARR being larger than that under offDARR (Table I), but not for C5 and C6. C5 and C6 are (a)

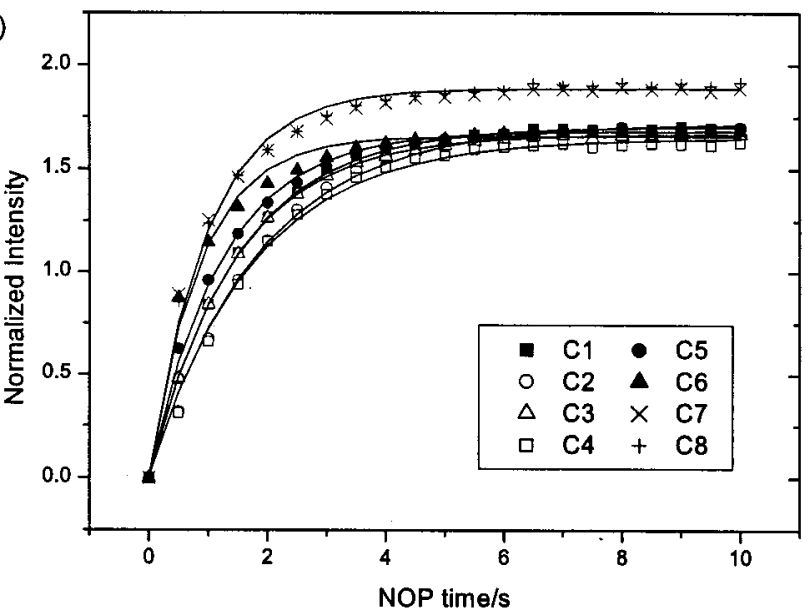

(b)

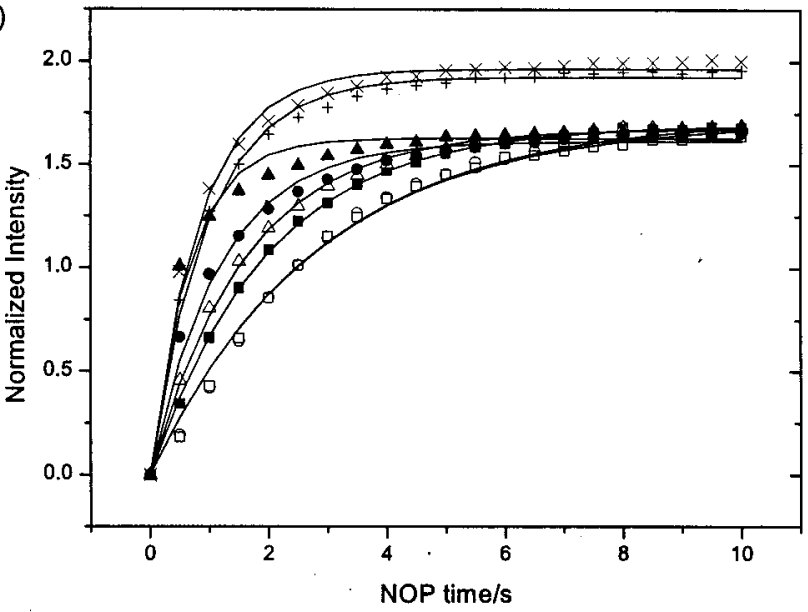

FIG. 4. NOP time dependence of the area intensities of the ${ }^{13} \mathrm{C}$ signals in uniformly ${ }^{13} \mathrm{C},{ }^{15} \mathrm{~N}$-labeled glycylisoleucine. DARR irradiation $\left(\nu_{1}=\nu_{\mathrm{MAS}}\right.$ $=20 \mathrm{kHz}$ ) was applied for (a), while $\nu_{1}=5 \mathrm{kHz}$ for (b). Each signal intensity is given by taking the corresponding area intensity observed by a ${ }^{13} \mathrm{C}$ $90^{\circ}$ pulse with a relaxation interval of $300 \mathrm{~s}$ as 1.0 . The solid lines are the best-fit curves to Eq. (10) with the best-fit parameters collated in Table I.

carbons bonded directly to the methyl carbons, C7 and C8, respectively, and thus direct $\mathrm{NOE}$ enhancement from ${ }^{1} \mathrm{H}$ in the methyl groups would occur in addition to NOP. Hence for C5 and C6, acceleration of $k_{\mathrm{NOP}}$ under DARR is not appreciable. For C6 in particular, we found that the C6-C8 bond apparently shows an unusual bond length, which may be caused by a large thermal vibration of $\mathrm{C} 8 .{ }^{13}$ Further, the observed ${ }^{13} \mathrm{C} T_{1}$ of $\mathrm{C} 6$ is as short as those of the methyl carbons (Fig. 3). Hence for C6, direct $\mathrm{NOE}$ from the $\mathrm{C}(6) \mathrm{H}_{2}$ protons as well as NOE due to fluctuation of the $\mathrm{C} 6-\mathrm{C} 8$ dipolar interaction should also contribute to $S(t)$ of C6. Thus omitting C6, we show the correlation between $k_{\mathrm{NOP}}$ and $r_{\mathrm{C}-\mathrm{C} 7}$ in Fig. 5. The observed correlation indicates a possibility of determining internuclear distances roughly by the polarization transfer experiment under DARR, which is currently underway and will be published elsewhere. Here we would like to point out that for $r_{\mathrm{C}-\mathrm{C} 7} \geqslant 0.5 \mathrm{~nm}$ intermolecular NOP becomes appreciable, thus leading $k_{\mathrm{NOP}}$ being insensitive to $r_{\mathrm{C}-\mathrm{C} 7}$. Hence for deducing structural information from the polarization transfer experiment under DARR, di- 
TABLE I. Best-fit parameters to the NOP-time dependence data in Fig. 4 and the $\mathrm{C}-\mathrm{C}$ distances from the $\mathrm{CH}_{3}$ carbons $(\mathrm{C} 7, \mathrm{C} 8)$.

\begin{tabular}{|c|c|c|c|c|c|c|c|c|c|c|}
\hline & \multicolumn{4}{|c|}{ On DARR [Fig. 4(a)] } & \multicolumn{4}{|c|}{ Off DARR [Fig. 4(b)] } & \multicolumn{2}{|c|}{ Distance/nm } \\
\hline & $\eta_{\mathrm{NOP}}$ & $\sigma$ & $k_{\mathrm{NOP}} / \mathrm{s}^{-1}$ & $\sigma$ & $\eta_{\mathrm{NOP}}$ & $\sigma$ & $k_{\mathrm{NOP}} / \mathrm{s}^{-1}$ & $\sigma$ & $r_{\mathrm{C}-\mathrm{C} 7}$ & $r_{\mathrm{C}-\mathrm{C} 8}$ \\
\hline $\mathrm{C} 1$ & 1.704 & 0.002 & 0.677 & 0.004 & 1.698 & 0.004 & 0.500 & 0.004 & 0.287 & 0.496 \\
\hline $\mathrm{C} 2$ & 1.719 & 0.011 & 0.546 & 0.014 & 1.737 & 0.022 & 0.348 & 0.010 & 0.496 & 0.560 \\
\hline C3 & 1.669 & 0.002 & 0.700 & 0.003 & 1.678 & 0.006 & 0.610 & 0.010 & 0.255 & 0.391 \\
\hline $\mathrm{C} 4$ & 1.650 & 0.012 & 0.573 & 0.017 & 1.718 & 0.021 & 0.353 & 0.012 & 0.619 & 0.639 \\
\hline C5 & 1.684 & 0.006 & 0.814 & 0.016 & 1.617 & 0.012 & 0.836 & 0.034 & 0.145 & 0.241 \\
\hline C6 & 1.658 & 0.012 & 1.158 & 0.056 & 1.630 & 0.015 & 1.490 & 0.105 & 0.250 & 0.164 \\
\hline $\mathrm{C} 7$ & 1.846 & 0.012 & 1.078 & 0.038 & 1.966 & 0.012 & 1.173 & 0.046 & 0.0 & 0.289 \\
\hline C8 & 1.846 & 0.011 & 1.078 & 0.041 & 1.927 & 0.009 & 1.024 & 0.030 & 0.289 & 0.0 \\
\hline
\end{tabular}

lution of the fully ${ }^{13} \mathrm{C}$-labeled sample by a natural abundance one should be used.

Table I shows that the NOP enhancement factors for the two $\mathrm{CH}_{3}$ carbons $\left(\eta_{\mathrm{NOP}} \sim 1.9\right)$ are appreciably larger than those of the other carbons $\left(\eta_{\mathrm{NOP}} \sim 1.7\right)$. Further, the nonmethyl carbons show the same $\eta_{\mathrm{NOP}}$ for the two experiments in spite of the different equilibration rates, while the $\mathrm{CH}_{3}$ carbons have slightly smaller $\eta_{\text {NOP }}$ under DARR. Putting $N=3$ for Gly-Ile (two methyl carbons and six non-methyl carbons) and $T_{1}^{L}=10 \mathrm{~s}, T_{1}^{S}=0.25 \mathrm{~s}$, and $k=1 \mathrm{~s}^{-1}$, which are estimated roughly from Fig. 3(a), into Eq. (8), we have $\eta_{\mathrm{NOP}}^{S} \sim 1+0.94 \xi_{\mathrm{NOE}}$ and $\eta_{\mathrm{NOP}}^{L} \sim 1+0.85 \xi_{\mathrm{NOE}}$, which explains qualitatively the observed difference in the apparent enhancement factors for the $\mathrm{CH}_{3}$ carbons $(\sim 1.9)$ and the other carbons $(\sim 1.7)$. Further, the observed reduction of $\eta_{\text {NOP }}^{S}$ of the two $\mathrm{CH}_{3}$ carbons under DARR can be ascribed to the increase of the polarization transfer rate $k$ due to DARR.

The observed enhancement factors of $\eta_{\mathrm{NOP}} \sim 1.9$ for the two $\mathrm{CH}_{3}$ carbons $(\mathrm{C} 7$ and $\mathrm{C} 8$ ) are significantly smaller than the maximum factor of 2.99. The smaller enhancement would be due partially to the polarization loss by the transfer to the other carbons [Eq. (8)] and mainly to a long $\mathrm{CH}_{3}$ rotational correlation time not to fulfil the extremely narrow-

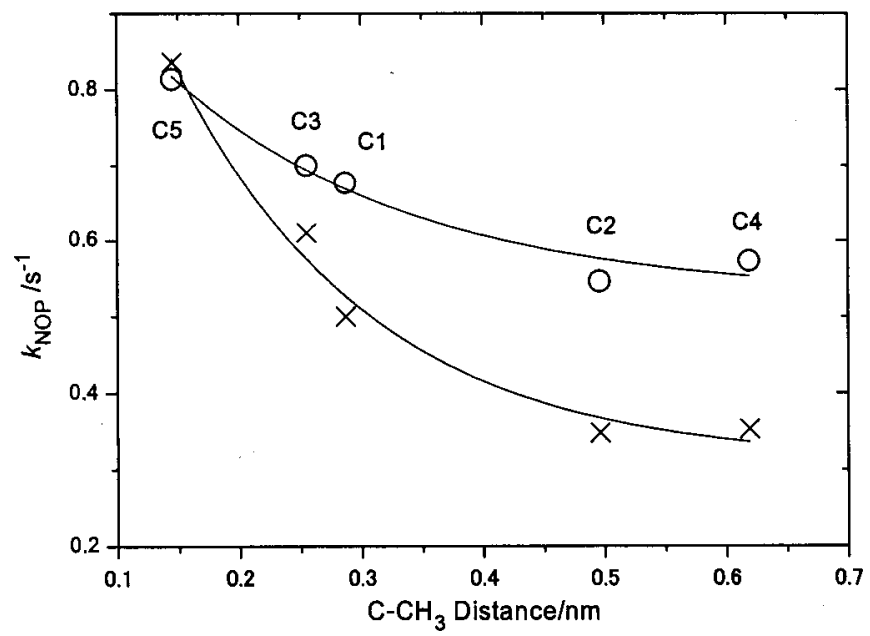

FIG. 5. Correlation between the apparent NOP buildup rate and the $\mathrm{C}-\mathrm{C}$ distance to the $\mathrm{CH}_{3}$ carbons (C7) for $\mathrm{C} 1-\mathrm{C} 5$ in uniformly ${ }^{13} \mathrm{C},{ }^{15} \mathrm{~N}$-labeled glycylisoleucine; open circles denote data under DARR irradiation $\left(\nu_{1}\right.$ $\left.=\nu_{\mathrm{MAS}}=20 \mathrm{kHz}\right)$ and crosses indicate data under off-DARR $\left(\nu_{1}\right.$ $=6 \mathrm{kHz}$ ). The solid lines are for eye guidance. ing condition. In fact, Naito et al. ${ }^{7}$ successfully reproduced their observed $\eta_{\mathrm{NOE}}$ value of 1.68 for the $\mathrm{CH}_{3}$ carbon in $L$-alanine by calculation with the literature rotational correlation time $\tau_{r}$ of $1.6 \times 10^{-9} \mathrm{~s}$. Unfortunately the ${ }^{1} \mathrm{H} T_{1}$ curve for L-isoleucine has not been fully analyzed. Hence, we estimated $\tau_{r}$ to be $\sim 7 \times 10^{-10}$ from $\eta_{\mathrm{NOE}} \sim 1.9$ using Eq. (2). The $\tau_{r}$ value is comparable to the $\tau_{r}$ values at $300 \mathrm{~K}$ for the $\mathrm{CH}_{3}$ groups in some other amino acids: ${ }^{18} \tau_{r}=1.2 \times 10^{-9} \mathrm{~s}$ for $L$-alanine, $1.2 \times 10^{-10} \mathrm{~s}$ for $D L$-leucine, $2.5 \times 10^{-11} \mathrm{~s}$ for $D L$-norleucine, $6.1 \times 10^{-11} \mathrm{~s}$ for $L$-threonine, and 1.8 $\times 10^{-10} \mathrm{~s}$ for $L$-valine.

Quantitativity of ${ }^{13} \mathrm{C}$ intensities is examined by comparing the area intensities observed for Gly-Ile using a ${ }^{13} \mathrm{C} 90^{\circ}$ pulse without NOP (the relaxation interval was $300 \mathrm{~s}$ ), with NOP (the NOP time was $6 \mathrm{~s}$ ) and using CP (the CP time was $2.6 \mathrm{~ms}$ and the relaxation interval was $4 \mathrm{~s}$ ). For the $90^{\circ}$ pulse experiment without NOP, the observed area intensities for the individual carbons are expressed in values relative to the average area intensity for the eight carbons. They are almost equal to unity with the small standard deviation $\sigma=0.035$, showing good uniformity for the relaxation interval of $300 \mathrm{~s}$. The observed signal intensities by NOP and CP for the individual carbons were normalized by the corresponding intensities for the $90^{\circ}$ pulse experiment and collated in Table II together with the averages and the standard deviations $\sigma$. For the $90^{\circ}$ pulse experiment with NOP, the standard deviation calculated for the eight carbons is somewhat larger: $\sigma$ $=0.098$. However, as indicated in Eq. (8), the different enhancement factors for the $\mathrm{CH}_{3}$ carbons and the other carbons should be taken into account. The average and the standard deviation obtained for the $\mathrm{C} 1-\mathrm{C} 6$ carbons are 1.65 and $\sigma$ $=0.022$, respectively, showing excellent uniformity achieved by NOP. For the CP spectrum, the standard deviation is the largest ( $\sigma=0.237$ ), showing that care must be taken to compare the intensities obtained by $\mathrm{CP}$ quantitatively. The larger variation found for $\mathrm{CP}$ is attributed to the differences of the ${ }^{13} \mathrm{C}$ spin-lattice relaxation times in the rotating frame and the $\mathrm{CP}$ rates among the carbons.

Figure 6 shows the DARR rf-strength dependence of the signal intensities observed for Gly-Ile with the NOP time of $5 \mathrm{~s}$ and $\nu_{\mathrm{MAS}}=20 \mathrm{kHz}$, demonstrating that in the polarization transfer experiment under DARR, a slight misadjustment of the ${ }^{1} \mathrm{H}$ rf intensity or the spinning frequency from the DARR condition is not crucial; $\pm 2 \mathrm{kHz}$ misadjustment from the DARR condition $\left(\nu_{1}=\nu_{\mathrm{MAS}}=20 \mathrm{kHz}\right)$ causes only 
TABLE II. Normalized area intensities of the eight carbons of uniformly ${ }^{13} \mathrm{C},{ }^{15} \mathrm{~N}$-labeled glycylisoleucine. ${ }^{\mathrm{a}}$

\begin{tabular}{lcccccccccc}
\hline \hline \multicolumn{1}{c}{ Method } & $\mathrm{C} 1$ & $\mathrm{C} 2$ & $\mathrm{C} 3$ & $\mathrm{C} 4$ & $\mathrm{C} 5$ & $\mathrm{C} 6$ & $\mathrm{C} 7$ & $\mathrm{C} 8$ & Average & $\sigma$ \\
\hline Without NOP & 1.03 & 1.07 & 0.99 & 1.01 & 0.97 & 0.98 & 1.00 & 0.95 & 1.00 \\
With NOP & 1.67 & 1.66 & 1.64 & 1.61 & 1.67 & 1.67 & 1.87 & 1.88 & 1.71 & 0.035 \\
CP & 1.78 & 1.99 & 2.02 & 1.99 & 2.17 & 2.29 & 2.50 & 2.47 & 2.15 & 0.237 \\
\hline \hline
\end{tabular}

${ }^{a}$ For the ${ }^{13} \mathrm{C} 90^{\circ}$ pulse experiment without NOP (the first row in the table), the area intensity of each carbon signal was expressed in values relative to the average of the area intensities of the eight carbon signals. For the ${ }^{13} \mathrm{C} 90^{\circ}$ pulse experiment with NOP (the second row in the table) and the CP experiment (the last row in the table), the area intensity for each carbon signal was normalized by the corresponding area intensity in the ${ }^{13} \mathrm{C} 90^{\circ}$ pulse spectrum without NOP.

a few percent change in intensity. However, the increase of the intensities of the $\mathrm{CH}_{3}$ carbons $(\mathrm{C} 7, \mathrm{C} 8)$ and the decrease of the other intensities particularly the glycine carbons $(\mathrm{C} 2$, C4) are notable at rf intensities far from the DARR condition. This tendency can also be confirmed in Fig. 4, and as have pointed out, can be explained by Eq. (8) with a slow polarization transfer rate $k$ at off-DARR conditions.

It would be interesting to examine whether NOP is applicable to natural-abundance samples. Figure 7 shows the ${ }^{13} \mathrm{C}$ MAS spectra observed for nonlabeled dimedone (5,5dimethyl-1,3-cyclohexanedione) using a ${ }^{13} \mathrm{C} 90^{\circ}$ pulse without (a),(b) and with NOP (NOP time of $10 \mathrm{~s}$ ) (c). The relaxation interval without NOP [Fig. 7(b)] was chosen to be $10 \mathrm{~s}$ to compare with the NOP spectrum [Fig. 7(c)], and that for the spectrum in Fig. 7(a) was $300 \mathrm{~s}$ to obtain a fully relaxed spectrum. The signals $\mathrm{C} 7$ and $\mathrm{C} 8$ are assigned to the $\mathrm{CH}_{3}$ carbons, ${ }^{21}$ and their NOP enhancement factors are $\approx 3.0$, reaching the theoretical maximum. Interestingly, we found that the signals for the other rigid carbons $(\mathrm{C} 1-\mathrm{C} 6)$, especially for the quarternary carbon (C5), do also show significant enhancement. Two signal enhancement mechanisms can be invoked, namely, direct NOE enhancement of the ${ }^{13} \mathrm{C}$ signals $(\mathrm{C} 1-\mathrm{C} 6)$ by the ${ }^{1} \mathrm{H}$ spins in the two $\mathrm{CH}_{3}$ groups and the ${ }^{13} \mathrm{C}-{ }^{13} \mathrm{C}$ polarization transfer from the two ${ }^{13} \mathrm{CH}_{3}$ spins. The large enhancement for $\mathrm{C} 5$, which is bonded directly to the two methyl carbons, supports the former mechanism. To ex-

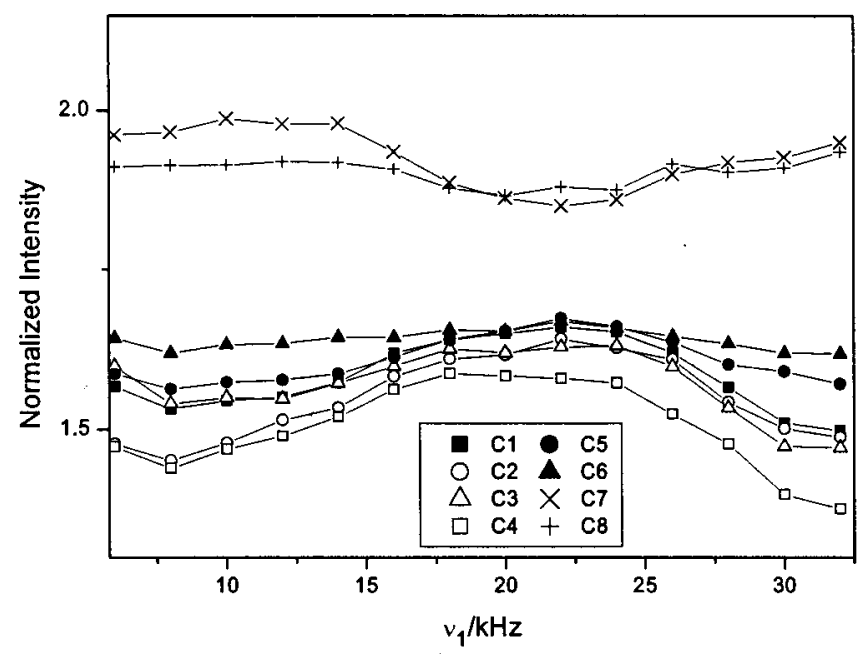

FIG. 6. DARR rf-strength dependence of the intensities of the signals in uniformly ${ }^{13} \mathrm{C},{ }^{15} \mathrm{~N}$-labeled glycylisoleucine at $\nu_{\mathrm{MAS}}=20 \mathrm{kHz}$. Each signal is normalized to the corresponding area intensity observed by a ${ }^{13} \mathrm{C} 90^{\circ}$ pulse with a relaxation interval of $300 \mathrm{~s}$. The solid lines are for eyeguidance. amine the latter, a $90^{\circ}$ pulse spectrum was recorded after ${ }^{1} \mathrm{H}$ irradiation for $10 \mathrm{~s}$ with the ${ }^{1} \mathrm{H}$ rf intensity of $5 \mathrm{kHz}$ (not shown), which is deviated significantly from the DARR condition $\left(\nu_{1}=\nu_{\mathrm{MAS}}=10 \mathrm{kHz}\right)$. We observed slight decreases in the $\mathrm{C} 1-\mathrm{C} 6$ intensities compared to that in Fig. 7(c). This reduction is similar to that observed in Fig. 4, showing that the latter ${ }^{13} \mathrm{C}-{ }^{13} \mathrm{C}$ polarization transfer mechanism takes place even for naturally abundant ${ }^{13} \mathrm{C}$ spins.

\section{CONCLUDING REMARKS}

We have shown that DARR irradiation causes NOE enhancement of ${ }^{13} \mathrm{CH}_{3}$ carbon signals and distributes the polar-

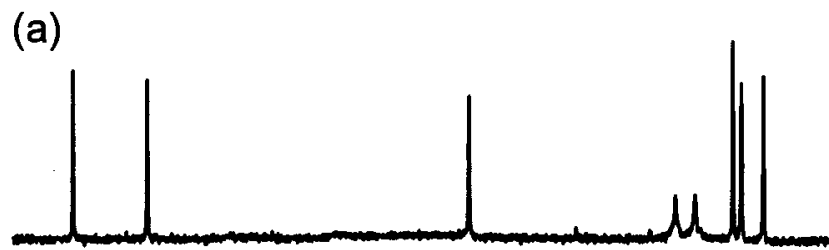

(b)

(c)
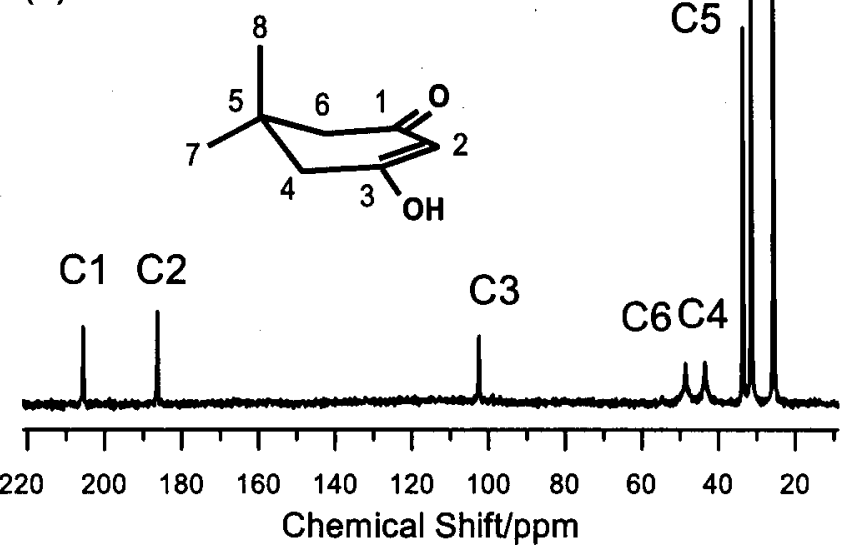

FIG. 7. ${ }^{13} \mathrm{C}$ MAS spectra obtained for nonlabeled dimedone (5,5-dimethyl1,3 -cyclohexanedione) by a ${ }^{13} \mathrm{C} 90^{\circ}$ pulse without (a),(b) and with NOP (c) under $\nu_{\text {MAS }}=10 \mathrm{kHz}$. The relaxation interval was (a) $300 \mathrm{~s}$ and (b) $10 \mathrm{~s}$. For the NOP enhancement (c), ${ }^{1} \mathrm{H}$ irradiation with $\nu_{1}=\nu_{\mathrm{MAS}}=10 \mathrm{kHz}$ was applied for 10 s. 256 FIDs were accumulated for each experiment, and the spectra are plotted on the same amplitude scale and can be directly compared. 
ization uniformly over all the carbons in a uniformly ${ }^{13} \mathrm{C}$ labeled system with mobile parts. Since many amino acids have mobile parts such as the $\mathrm{CH}_{3}$ groups, the present approach is applicable to most of uniformly/segmentally isotope-labeled peptide samples. Such extensive ${ }^{13} \mathrm{C}$ labeling has been becoming an important method for solid NMR to elucidate structural information of biomolecules such as peptides and proteins. For a protein molecule, it is expected that mobility of sidechains would increase due to weaker crystallographical packing force. For example, from the ${ }^{1} \mathrm{H} T_{1}$ data of Streptomyces subtilisin inhibitor (SSI), ${ }^{22}$ we can obtain the correlation time of the motion to be $9.6 \times 10^{-12} \mathrm{~s}$ at $300 \mathrm{~K}$, indicating that the extreme narrowing limit of relaxation is achieved thus leading to the maximum NOP enhancement for SSI. Similar results can be deduced for ribonuclease A, $\alpha$-chymotrypsin, and lysozyme. ${ }^{23}$

In uniformly ${ }^{13} \mathrm{C}$ labeled peptides, due to the small number ratio of ${ }^{1} \mathrm{H}$ to ${ }^{13} \mathrm{C}$ nuclei, the signal enhancement factor achieved by $\mathrm{CP}$ becomes $\sim 2.4$, which would practically be reduced because of imperfect spin-locking by phase transient and the effect of finite $T_{1 \rho}$ of ${ }^{1} \mathrm{H}$ and ${ }^{13} \mathrm{C}$. On the other hand, NOP provides the factor of 3 provided that the rotational correlation times of the $\mathrm{CH}_{3}$ groups are in the extreme narrowing region. NOP enables us to acquire a signal with a short repetition time even if ${ }^{1} \mathrm{H} T_{1}$ is long. For molecules with short $T_{1 \rho}$ of ${ }^{1} \mathrm{H}$ or ${ }^{13} \mathrm{C}$, the $\mathrm{CP}$ enhancement becomes smaller, while NOP would work well for such mobile molecules. Furthermore, the present DARR approach is insensitive to rf inhomogeneity and to missetting and fluctuation in $\nu_{\mathrm{MAS}}$ and $\mathrm{H}_{1}$, and can easily be carried out with low rf power. Moreover, NOP can be used for quantitative analysis of ${ }^{13} \mathrm{C}$ signals in solids, while it is nearly impossible by $\mathrm{CP}$. Lastly, NOP may be used to enhance NMR signals of nuclei with low $\gamma$, such as, ${ }^{57} \mathrm{Fe},{ }^{107} \mathrm{Ag}$, etc. in a mobile molecule or a molecule having a mobile group, while $\mathrm{CP}$ cannot readily be applied because of the necessity of very high rf power in case a certain pulse technique such as TAPF (Ref. 24) is not incorporated.

\section{ACKNOWLEDGMENT}

This research was supported by a Grant-in-Aid for Science Research from the Ministry of Education, Culture, Sports, Science, and Technology of Japan.

${ }^{1}$ S. R. Hartmann and E. L. Hahn, Phys. Rev. 128, 2042 (1962).

${ }^{2}$ A. Pines, M. G. Gibby, and J. S. Waugh, J. Chem. Phys. 59, 569 (1973).

${ }^{3}$ A. W. Overhauser, Phys. Rev. 91, 476 (1953); 92, 411 (1953).

${ }^{4}$ E. G. Paul and D. M. Grant, J. Am. Chem. Soc. 86, 2977 (1964).

${ }^{5}$ F. A. L. Anet and A. J. R. Bourne, J. Am. Chem. Soc. 87, 5250 (1965).

${ }^{6}$ For examples, see, D. T. Okamoto, S. L. Cooper, and T. W. Root, Macromolecules 25, 3301 (1992); J. L. White and P. Mirau, ibid. 26, 3049 (1993).

${ }^{7}$ A. Naito, S. Ganapathy, K. Akasaka, and C. A. McDowell, J. Magn. Reson. 54, 226 (1983); A. Naito and C. A. McDowell, J. Chem. Phys. 84, 4181 (1986).

${ }^{8}$ K. Takegoshi, S. Nakamura, and T. Terao, Chem. Phys. Lett. 344, 631 (2001).

${ }^{9}$ T. G. Oas, R. G. Griffin, and M. H. Levitt, J. Chem. Phys. 89, 692 (1988).

${ }^{10}$ E. R. Andrew, S. Clough, L. F. Farnell, T. D. Gledhill, and I. Roberts, Phys. Lett. 21, 505 (1966).

${ }^{11}$ D. P. Raleigh, G. S. Harbison, T. G. Neiss, J. E. Roberts, and R. G. Griffin, Chem. Phys. Lett. 138, 285 (1987).

${ }^{12}$ B. H. Meier and W. L. Earl, J. Am. Chem. Soc. 109, 7939 (1987).

${ }^{13}$ K. Nomura, K. Takegoshi, T. Terao, K. Uchida, and M. Kainosho, J. Biolmol. NMR 17, 111 (2000).

${ }^{14}$ A. E. Bennett, C. M. Rienstra, M. Auger, K. V. Lakshmi, and R. G. Griffin, J. Chem. Phys. 103, 6951 (1995).

${ }^{15}$ D. A. Torchia and A. Szabo, J. Magn. Reson. 49, 107 (1982).

${ }^{16}$ I. Solomon, Phys. Rev. 99, 559 (1955).

${ }^{17}$ A. A. Brooks, J. D. Cutnell, E. O. Stejskal, and V. W. Weiss, J. Chem. Phys. 49, 1571 (1968).

${ }^{18}$ E. R. Andrew, W. S. Hinshaw, M. G. Hutchins, and R. O. I. Sjöblom, Mol. Phys. 32, 795 (1976).

${ }^{19}$ J. E. Anderson and W. P. Slichter, J. Phys. Chem. 69, 3099 (1965).

${ }^{20}$ U. Haeberlen, Philos. Trans. R. Soc. London, Ser. A 299, 497 (1981).

${ }^{21}$ F. Imashiro, S. Maeda, K. Takegoshi, T. Terao, and A. Saika, Chem. Phys. Lett. 92, 642 (1982).

${ }^{22}$ K. Akasaka, K. Takegoshi, T. Terao, and S. Ganapathy, Can. J. Chem. 66, 2014 (1988)

${ }^{23}$ E. R. Andrew, D. J. Bryant, and E. M. Cashell, Chem. Phys. Lett. 69, 551 (1980).

${ }^{24}$ K. Takegoshi and C. A. McDowell, J. Magn. Reson. 67, 356 (1986). 